\title{
Performance Analysis of an OFDM PHY Scheme with Zero Forcing Equalizer Using Software Defined Radio Platform and USRP
}

\author{
Ahmad Zainudin, Amang Sudarsono, I Gede Puja Astawa \\ Postgraduate Applied Engineering of Technology \\ Division of Electrical Engineering, Dept. of Electrical Engineering \\ Electronic Engineering Polytechnic Institute of Surabaya (EEPIS) \\ Kampus PENS, Jalan Raya ITS, Sukolilo 60111, Indonesia \\ Email : \{zai, amang, puja\}@pens.ac.id
}

\begin{abstract}
We present an implementation of Zero Forcing (ZF) equalizer in OFDM scheme using Software Defined Radio platform whereas NI USRP-2920 as the Radio Frequency (RF) front-end. ZF equalizer is employed to achieve reliable system at the receiver. Center frequency used for data transmission is $915 \mathrm{MHz}$. The reliability transmission and the performance of ZF equalizer are measured in term of different symbol mapping (i.e., M-PSK and M-QAM). The IQ rate determines the bandwidth available, whereas good performance is achieved with IQ rate less than $1 \mathrm{MHz} . \mathrm{ZF}$ equalizer achieves good performance when using BPSK, QPSK and 16-QAM modulation techniques. By applying ZF equalizer, bit error on BPSK and QPSK modulations can be reduced from $29,16 \%$ and $39,06 \%$ into $0 \%$. This advantage of ZF equalizer also is able to press the bit error on 16QAM and 64-QAM modulations into 3,125\% and 8,85\%, respectively.
\end{abstract}

Keywords: OFDM,SDR, USRP,Zero Forcing Equalizer

\section{INTRODUCTION}

Recently, demand of bandwidth is increasing, especially for mobile devices. Orthogonal Frequency Division Multiplexing (OFDM) is introduced as one of solutions to enable bandwidth efficiency and robustness due to intersymbol inteference (ISI) in the consequence of multipath fading environment [1]. The principle of OFDM is dividing high rate data stream into parallel low rate data streams using Fast Fourier Transform (FFT)[2]. Moreover, OFDM scheme is widely used in wireless communication system, such as IEEE 802.11 standard, cellular communication (e.g., WiMAX and LTE Advanced) and digital broadcasting standard (e.g., DVB-T). In addition, OFDM is combined by modulation with more bits per symbol to increase data 
transmission throughput. OFDM has been successfuly implemented in wireless communication due to its advantages of reliability communication in multipath environment. However, OFDM system has the problem in dealing with ISI effect particularly for the high data rate transmission.An equalizer is required to combat intersymbol inteference when OFDM symbol time is larger than delay spread. The implementation of equalizer requires more hardware complexity. The used of Software Defined Radio (SDR) can reduce hardware investment, due to the hardware implementation is changed into a software configuration.

SDR is a choice of wireless implementation because of its easier, faster and cheaper implementation favored. SDR is able to change hardware components (i.e., modulator, demodulator, filter, detector, equalizer, multiplexing/demultiplexing, and amplifier) into a software configuration in personal PC or embedded system. This benefit is able to decrease hardware investment along with the complexity of wireless technologies.SDR comprises baseband processing, Analog to Digital Converter (ADC)/Digital to Analog Converter (DAC) and RF front end. Universal Software Radio Periperal (USRP) is front end part which can be used for good performance in wireless implementation. The main goal of SDR platform is not only cost reduction, but also increasing of the flexibility signal processing parameters [3].

In this paper, we introduce an implementation of ZF equalizer in OFDM system. The NI USRP-2920 tranceivers are used as RF front-end and LabVIEW SDR platform control system. The reliability system and the performance of ZF equalizer are observed. From experimental results, using ZF equalizer on BPSK and QPSK modulations can reduce bit error from $29,16 \%$ and $39,06 \%$ into $0 \%$. This advantage also can press bit error on 16QAM and 64-QAM modulations into 3,125\% and 8,85\%, respectively.The layout of this paper is organized as follows, firstly, we describe the introduction of OFDM. Then, SDR and USRP are described in the first Section. In Section 2, the implementation OFDM is detail explained. The experimental results and analysis are presented in Section 3. And finally, our conclusion is drawn in Section 4.

\section{RELATED WORKS}

Currently, several studies of OFDM implementation using SDR and USRP have been taken in account. An OFDM system by using BPSK and QPSK modulation techniques and utilizing GNU Radio and USRP are implemented by [4-7] with good performance be achieved by QPSK rather than BPSK modulation.In these experiments are used basic OFDM system. Data streams still are generated from random bits source. The reliability system is ignored. In the [7] Bit Error Rate (BER) performance versus Signal to Noice Ratio (SNR) is compaired between Matlab simulation and real implementation experimental result. The performance result of simulation and implementation are not exactly the same. In the real experiment is difficult to 
measure the exact SNR value. The reliable transmission is offered [8] with employe Forward Error Correction (FEC). The performance of FEC is poor if the SNR lower than the threshold. Whereas, the noise environment are variants in real implementation system. The other algorithm is proposed [9] use a simple subcarrier blinding algorithm to compensate phase error. The worse performance is due to higher phase noise of the oscillator as internal reference clock. The performance of Zero Forcing equalizer with BPSK modulation is observed by [10] using matlab simulation. This equalizer can remove the effect of ISI when the channel is noiseless. The ZF equalizer performance is poor when the channel is noisly. ZF performance for single carrier and uncode OFDM is compared [11]. ZF-Single Carrier has better performance than the uncode OFDM.

\section{ORIGINALITY}

This paper presents a practical performance analysis of OFDM scheme. The OFDM transceiver is sucessfully constructed using LabVIEW platform and NI USRP-2920 as RF front-end. Reliability of the system is observed based on the symbol received constelation in the receiver side. The text file is sent over wireless medium with the $915 \mathrm{MHz}$. Moreover, a simple Zero Forcing (ZF) equalizer is employed on receiver side to combat for Inter Symbol Inteference (ISI). Unequalized and equalized data constellation are compared to determine equalizer performance used.

\section{SYSTEM DESIGN}

This system constructed OFDM scheme with employee Zero Forcing (ZF) equalizer in the receiver. Equalizer is used to combat ISI effect and can increase of reliable system. The algorithm is observed in real implementation system use USRP as RF front-end and SDR platform.

\subsection{Orthogonal Frequency Division Multiplexing (OFDM) Scheme}

OFDM is multiplexing scheme which divide data stream became more narrowband data channel to share the bandwidth available. Narrowband channel is called subcarrier which transmit phase or amplitude modulated data signal. Different with Frequency Division Multiplexing (FDM), OFDM have subcarrier that orthogonality. This orthogonality can reduce inteference between subcarrier and increase spectrum efficiency utilization. OFDM receiver require frequency synchonization to combat Inter-Carrier Interference (ICI).This inteference occurs caused by Doppler shift due the mobile device movement and multipath channel[2].

Data bits stream are converted from serial to parallel and each subcarrier is modulated using phase or amplitude modulation. Modulation prosses is called as symbol mapping. Each subcarrier is independently modulated using. Then, all the modulated signal are carried by OFDM carrier use IFFT module to create complex signal containing all subcarrier. Data parallel stream is converted to serial stream and real and imaginer signal 
respectively are processed on Digital to Analog Converter (DAC). Both analog signal are multiplied by radio frequency with shift it's phase $90^{\circ}$ and summing both. This signal will transmit over antenna. Figure 1 shows the diagram of OFDM system. The receiver will receive real and imaginer signal separately and they are processed by low pass filter to eliminate mirrored frequencies. Then, they are quantized by Analog to Digital Converter (ADC) block and the signal is calculated by Fast Fourier Transform (FFT) module. The data symbols are demodulated by symbol mapping block according to the modulation used. Parallel data stream is converted into data serial to obtain the desired data.

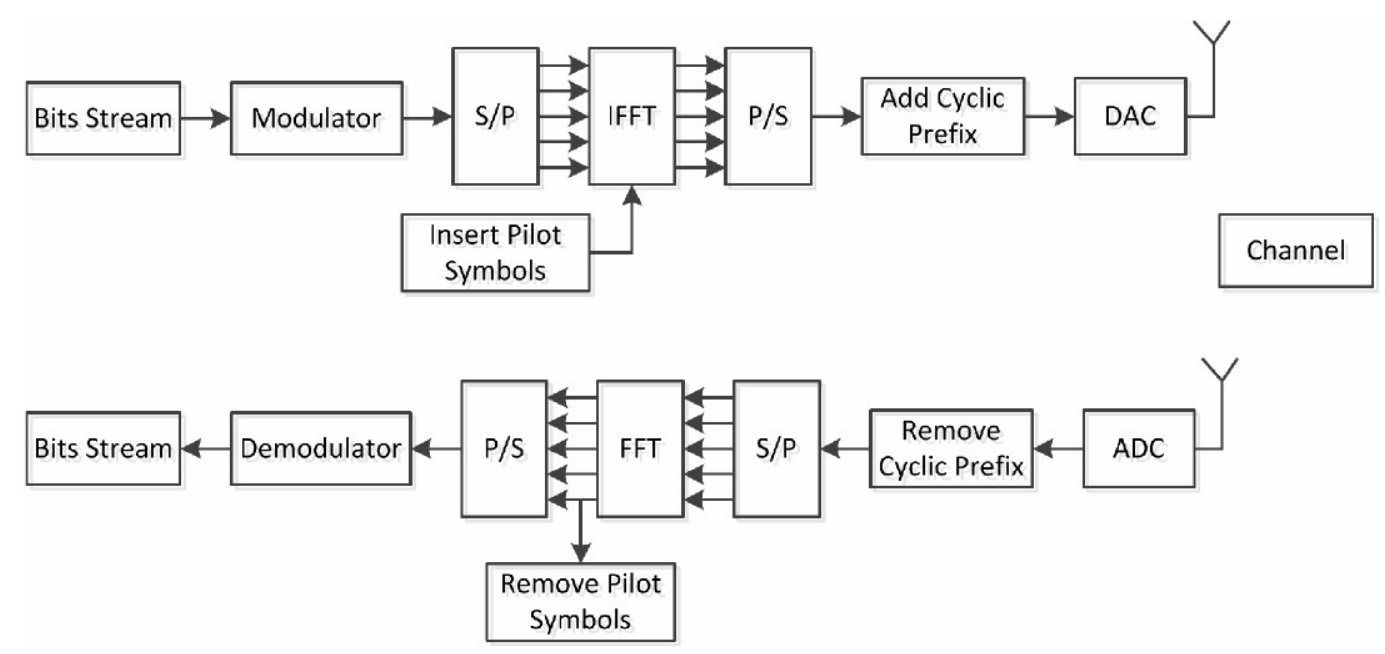

Figure 1.Block diagram of ofdm transmission system

Received signal will be received many times due multipath propagation specially in urban environment or mobile device are moving high speed. Line of sight and multipath signal have difference arrival time. It is called delay spread which can cause Inter Symbol Inteference (ISI). OFDM scheme proposes to reduce of ISI effect provided that can preserve orthogonality. Orthogonality OFDM subcarrier can be achieved with addition of guard time (guard interval). The OFDM guard time can be do by insert zero padding (ZP) or cyclic prefix (CP). CP is to extend the OFDM symbol by copying the last samples of the OFDM symbol into its front. CP is introduced before the OFDM symbol. Let $\mathrm{T}_{\mathrm{G}}$ denote the length of $\mathrm{CP}$ and $\mathrm{T}_{\text {sub }}$ denote the duration of OFDM symbol without guard time. So, the extended OFDM symbols now have the duration of $\mathrm{T}_{\mathrm{sym}}=\mathrm{T}_{\mathrm{sub}}+\mathrm{T}_{\mathrm{G}}$. Guard time is selected longer than multipath delay so as not to cause inteference with the next OFDM symbol. The other advantage of $\mathrm{CP}$ is combating Inter Carrier Inteference (ICI) which is crosstalk between subcarriers.

OFDM system needs synchronization in the receiver side to find the beginning of each symbols corectly. Synchronization parameters include finding the right time delay, frequency deviation and phase shift of each symbols in the subcarrier. These parameters can be determined with 
addition redundancy in some of the subcarriers which transsmitted. The redundancy is called pilot symbol or preamble. The parameters are known by looking pilot signal from data received and will be calculated for synchronization and channel estimation process. The density of pilots detemine quality of synchronization but decrease of data rate transmission.

\subsection{Zero Forcing (ZF) Equalizer}

Equalization is employed to combat inter symbol inteference which occur if OFDM symbol time is larger than delay spread. Equalizer is a filter that use an approximated inverse of channel frequency response or channel estimation[2]. ZF is a simple frequency domain equalizer can reduce of ISI effects, but it will greatly affected of inhancing the noise power. The ZF employ channel estimation $\left(H_{e s t}\right)$ and generally based on pilot subcarrier frequency. Let $s(n)$ denote the bits squence from text file and the $\hat{s}(n)$ denote the data estimation. The equation of ZF can expresed as

$$
\hat{\mathrm{s}}(n)=s(n) * \frac{1}{H_{\text {est }}}
$$

\subsection{Software Defined Radio (SDR)}

SDR is block communication system such as modulation, demodulation, filter, encoding and decoding, source coding, channel coding, ect, are provided as software code in the PC or embedded systems. These technologies help the researcher and provider to reduce the hardware investment to implement and examine the new standard communication or algorithm enhancement[12]. The implementation is more flexible, all radio paramaters are set through software configure for same radio device to be operated with any type of waveform. This contributes to development and research easier, faster and cheaper. There are several type SDR platform which widely implemented in the wireless communication research community, such as LabVIEW and opensource GNU Radio. LabVIEW is a data flow graphical programming language designed fo several application (i.e. control, measurement and RF communication)[13]. GNU Radio is an open source software for digital signal processing (DSP) function that was developed using C++ (low-level code) and python (high-levelcode) programming.SDR architecture for transmitter and receiver is illustrated as Figure 2.

For transmit the data computer host generate complex digital baseband signal. Wideband data converters cannot support the processing bandwidth and dynamic range required across different wireless standards. As a result, the analog-to-digital converter (ADC) and the digital-to-analog converter (DAC) are usually operated at intermediate frequency (IF). In digital IF block there are three processes before through the DAC block. The first DUC (Digital Upconverter) process which baseband signal is filtered and interpolated before it is quadrature modulated with a tunable carrier 
frequency. Second process is Crest Factor Reduction (CFR) and then Digital Predistortion (DPD) which is linearization techniques. High-speed mobile data versions employ non-constant envelope modulation techniques such as quadrature phase shift keying (QPSK) and quadrature amplitude modulation (QAM), so linearity is needed on the power amplifiers. Digital IF is converted to analog signal through DAC block. Then, the signal is multiplied by local oscillator and through Power Amplifier (PA) then transmitted over antenna.

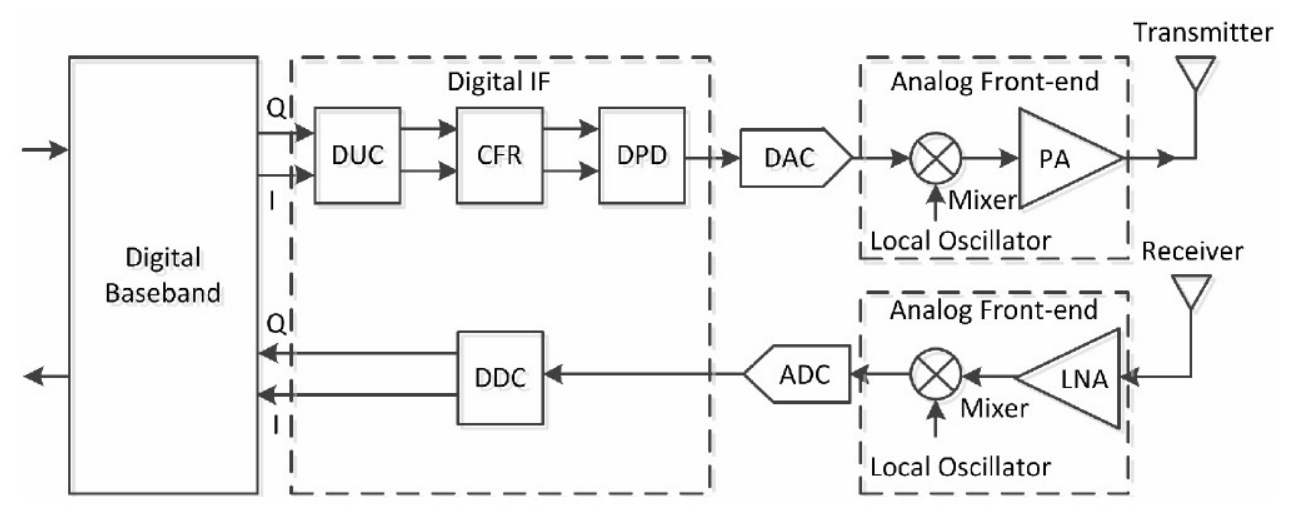

Figure 2.SDR Architecture for transmitter and receiver

\subsection{Universal Software Radio Peripheral (USRP)}

SDR implementation use USRP which hardware device that as a RF front end. USRP is now supported in Simulink and LabVIEW [14].The USRP consist of an FPGA, A/D and D/A, RF daugther board, and an interface to the host port. USRP devices are manufactured by Ettus Research and National Instrumen (NI). Ettus issue several of USRP's types, such as USRP bus serries (first generation), embedded serries and network serries (second generation). The first generation if USRPs use USB 2.0 connection with PC host which provides up to $\approx 30 \mathrm{MHz}$. But the second generation use gigabit ethernet up to $50 \mathrm{MHz}$ and is powered by a $6 \mathrm{VDC}[15]$. The second generation widely used for real time implementation wireless communication such as USRP2 and USRP N210. For So with same device can be used for several band frequencies.

Recently, NI has manufactured several USRP's types. USRP NI-2920 and USRP NI-2921 widely implemented with combining LabVIEW software. The NI-2920 can be used for several wireless applications including white space, broadcast FM, public safety, land-mobile, low-power unlicensed device (ISM) bands, sensor networks, cell phones, amateur radio and GPS. The NI USRP2920 has frequency range of $50 \mathrm{MHz}$ to 2,2 GHz. On other hand, USRP NI2921 has frequency range of 2.4 to $2.5 \mathrm{GHz}$ and 4.9 to $5.9 \mathrm{GHz}$, for implemention of low-power unlicensed devices on industrial, scientific and medical (ISM) bands at $2.45 \mathrm{GHz}$ or $5.8 \mathrm{GHz}[16]$. 


\section{IMPLEMENTATION}

OFDM PHY is contructed in this system. OFDM transceiver are implemented use NI USRP-2920 and LabVIEW platform. On transmitter side, the initalize process begins with open the session of USRP hardware. In this process is determined USRP IP's used. After that, configuring the signal such as determining IQ rate, carrier frequency, gain and active antenna. The next step is encodig the file source. Read from binary file function is used to read text file with the return string data form. String data is converted became byte array data. Byte array is unpacked to obtain bits 0's and 1's with decide 8 bits per integer. Bit stream 0's and 1's through mapper symbols appropriate modulation used. BPSK symbol consists 1 bit, 2 bits for QPSK, 4 bits form 16-QAM and 6 bits for 64-QAM. Mapping symbol is determined considered a type modulation scheme.

The OFDM symbols are constructed from 48 data symbols (48 pts per OFDM symbol). The pilot symbols are inserted after every $12^{\text {th }}$ data symbol (52 pts per OFDM symbol). Pilot symbols are used to handle channel estimation. Zero padding are placed at the edges ( 6 zeroes as start symbol and 5 zeroes as stop symbol respectively) and 1 zero at DC carrier (64 pts per OFDM symbol). Performe a Inverse FFT to convert the frequency domain signals to the time domain. Insert a $1 / 4$ cyclic prefix by duplicating the last 16 point OFDM symbol and are placed at beginning ( 80 pts per OFDM symbol). Then the digital signals are converted to analog signals. Transmit the signals using a USRP as RF front end. The transmission process can be seen at Figure 3 and 4.

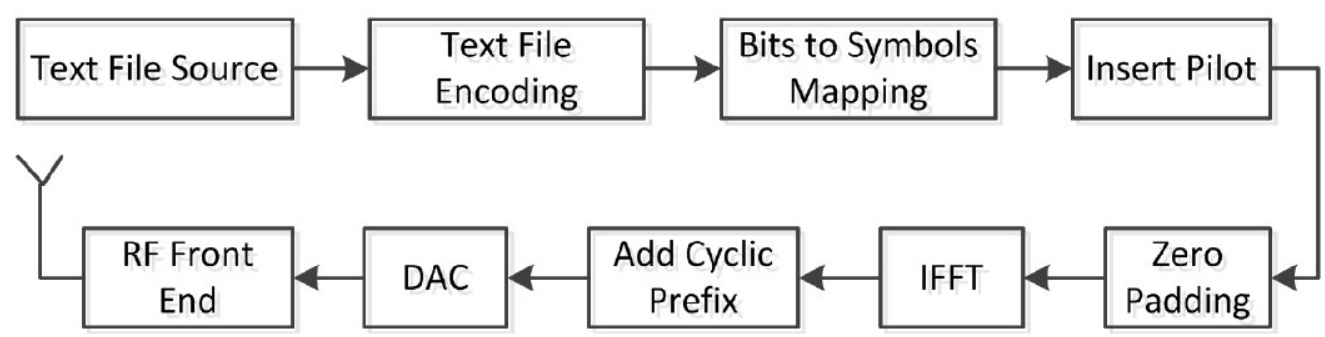

Figure 3. Transmission process at transmitter side

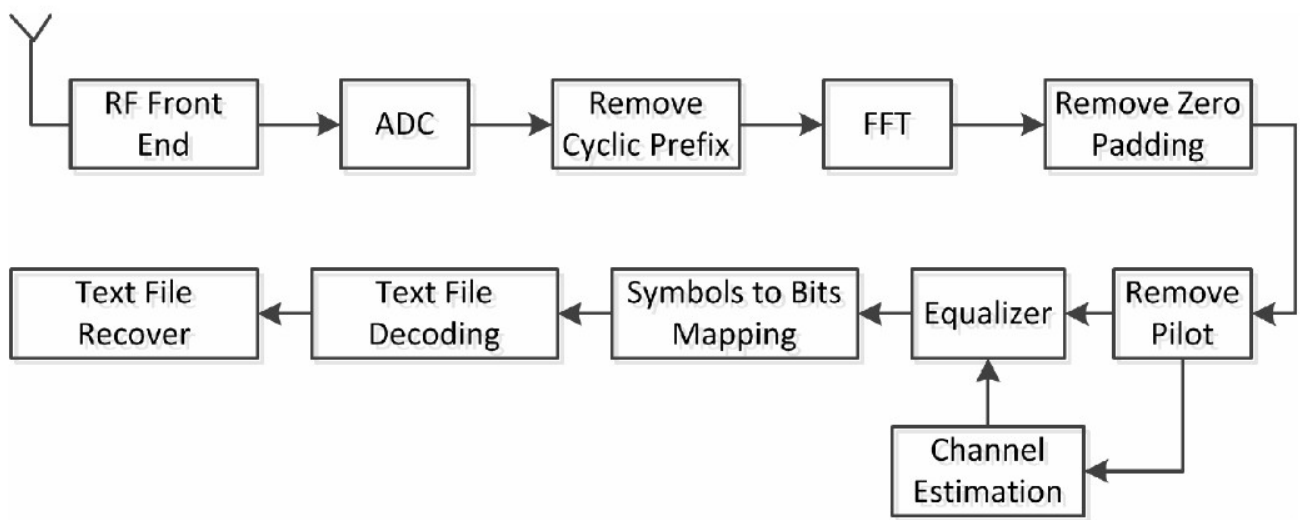

Figure 4. Transmission process at receiver side 
The signals are received and converted to digital signals. In this process begins USRP initalize with open Rx session and signal configure. USRP IP's, gain and active antenna are determined. IQ rate and carrier parameters are same with transmitter configure. The cyclic prefix and offset frequency are removed and the signals are converted to frequency domain through FFT process. Guard band (zero padding) are remove at OFDM symbol edges, respectively. Pilot symbols removing is used as channel estimation reference. The multiple of data and channel estimation invers will obtain equalized data. Bits stream be obtained after through symbols to bits mapping block. The sum of bits generated corresponding with modulation mapping used. The binary file is read and create text file use text file decoding. The OFDM parameters is shown at Table 1.

Table 1. Parameters of OFDM PHY design

\begin{tabular}{|l|c|}
\hline \multicolumn{1}{|c|}{ Parameter } & Value \\
\hline FFT size $\left(N_{F F T}\right)$ & 64 \\
\hline Number of data subcarrier & 48 \\
\hline Number of pilot subcarrier & 4 \\
\hline Number of start zero padding subcarrier & 6 \\
\hline Number of stop zero padding subcarrier & 5 \\
\hline Number of DC carrier subcarrier & 1 \\
\hline Cyclic prefix (CP) ratio & $1 / 4$ \\
\hline Number of total subcarrier & 80 \\
\hline Center frequency & $915 \mathrm{MHz}$ \\
\hline
\end{tabular}

\section{EXPERIMENT AND ANALYSIS}

The performance measurement of OFDM system which is constructed includes : the reliability of image transmission using several modulation (i.e BPSK, QPSK, 16-QAM and 64-QAM), the data rate transmission and the performance of Zero Forcing (ZF) equalizer is observed based on constellation diagram on the receiver side. The text file is sent with file size 1 $\mathrm{kB}$.

\subsection{The reliability of text file transmission measurement}

The purpose of this step to observe the reliability of OFDM system which cronstructed. The text file be sent from one NI USRP-2920 to another use $915 \mathrm{MHz}$ center frequency wit FFT length 64. The system is observed on indoor enviroment. The OFDM spectrum can be seen at Figure 5. The frequency band of the spectrum depending on In phase and Quadrature IQ rate used. IQ sampling rate is sampling frequency $\left(\frac{F_{S}}{2}\right)$ and determine of bandwidth avalible. In this experiment is used IQ rate $2 \mathrm{MHz}$. The figure shows that DC carrier is in the middle on carrier $\left(\frac{N}{2}\right)$ where $N$ is FFT length used. If the IQ rate is increased, spectrum bandwidth will go up too. Figure 
6shows the spectrum with different IQ rate. The greater of the IQ rate used, the computation of computer host is increasing too. This phenomenon will be felt when one computer host control several USRP (e.g. transmitter and receiver).

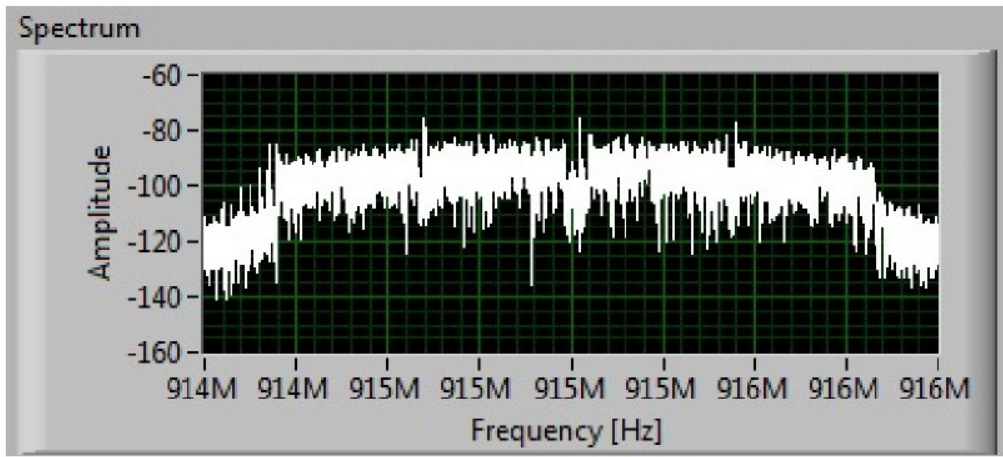

Figure 5. The spectrum of OFDM signal with IQ rate $2 \mathrm{MHz}$

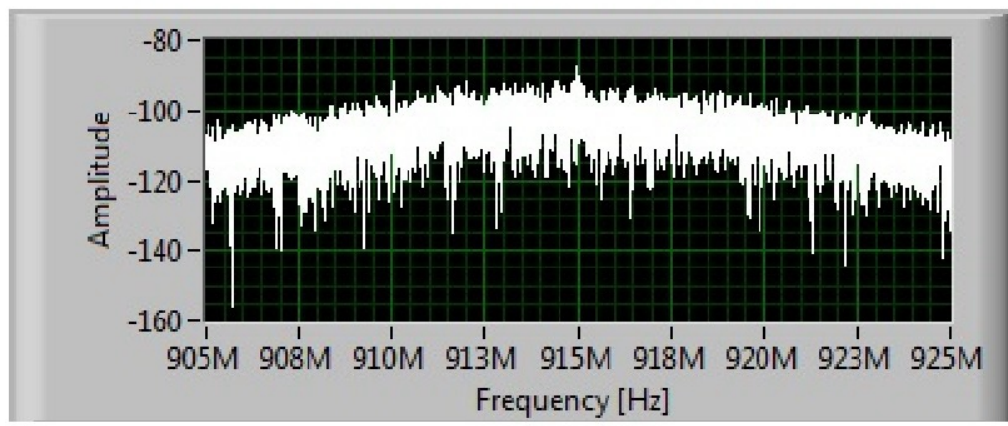

Figure 6. The spectrum of OFDM signal with IQ rate $20 \mathrm{MHz}$

The NI USRP-2920 has available bandwidth up to $40 \mathrm{MHz}$ [21]. The next step will be observed of comparison the IQ rate with the reliability of transmission. The IQ rate is increased and the data accuracy is observed based on constellation diagram. The Table 2 shows the constellation diagram with different IQ rate and are modulated using QPSK. The reliability system is achieved when use IQ rate less than $1 \mathrm{MHz}$. When the IQ rate is more than 1 $\mathrm{MHz}$, the error data which received increase. Although the NI USRP-2920 supporttransmits and receives bandwidth up to $40 \mathrm{MHz}$.

Table 2. Comparison of constellation diagram with different IQ rate

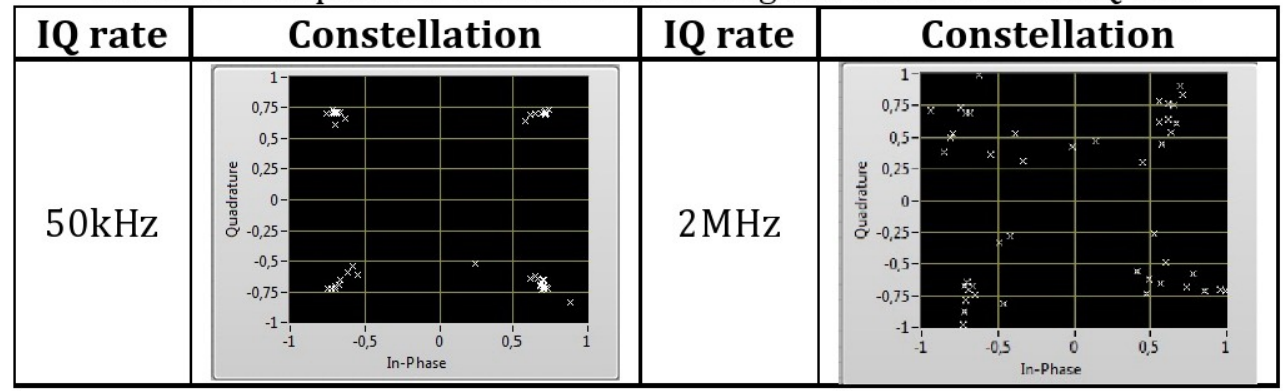




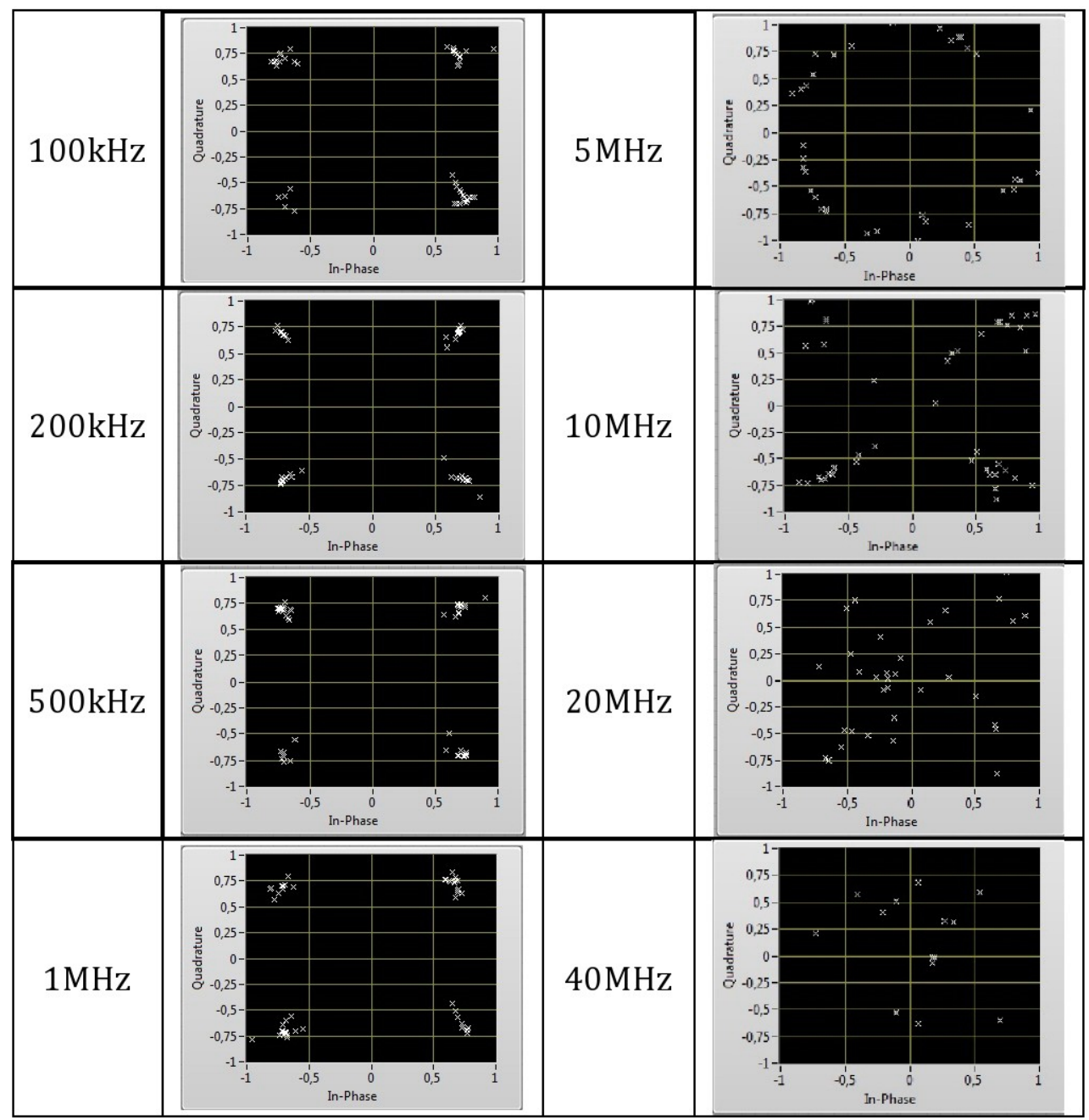

\subsection{The performance ofZero Forcing (ZF) equalizer}

The equalizer is used to combat ISI which making the communication less reliable. The performance of equalizer can be observed based on the accuracy of contellation diagram on the receiver side. In this experiment use IQ rate $200 \mathrm{kHz}$. Comparison of constellation diagram for several modulation is shown in Table 3. The good performance of ZF equalizer is achieved when is modulated using BPSK, QPSK and 16-QAM. The good performance is achieved because these modulation are resistant with noise. The poor performance occur when use 64-QAM. The more error data are received when this modulation is used. The performance system can be increasing with more complexity equalizer or non linear equalizer. 
Table 3. Comparison of constellation diagram using ZF equalizer for several modulation

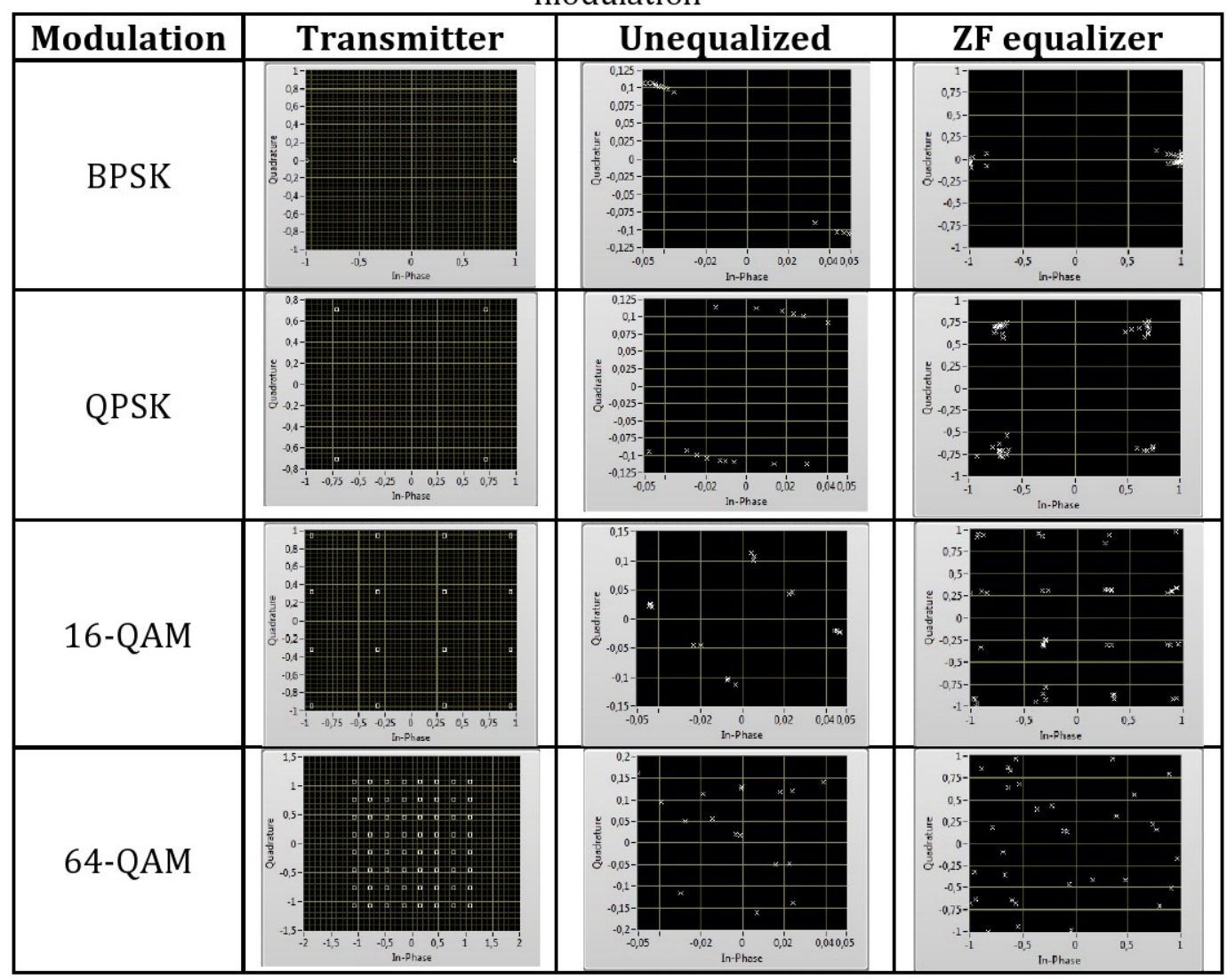

The ZF equalizer can be used for error correction of the data. The performance of ZF equalizer is also observed based on bit error of text file sent for several modulation. The bit error be obtained with comparing of text file source and text file recover. The compairing of bit error for unqualized data and use ZF equalizer with several modulation is shown at Figure 7. With use ZF equalizer can reduce bit error from 29,16\% into 0\% when use BPSK modulation. As well as when use QPSK modulation, bit error can be pressed with same value from 39,06\%. Whereas, the bit error began to increase when 16-QAM and 64-QAM modulation used, became 3,125\% and 8,85\%. But this error is not so large when compared with unequalized on same modulation. The bit error achieve $44,79 \%$ and $53,125 \%$ when equalizer is not used. 


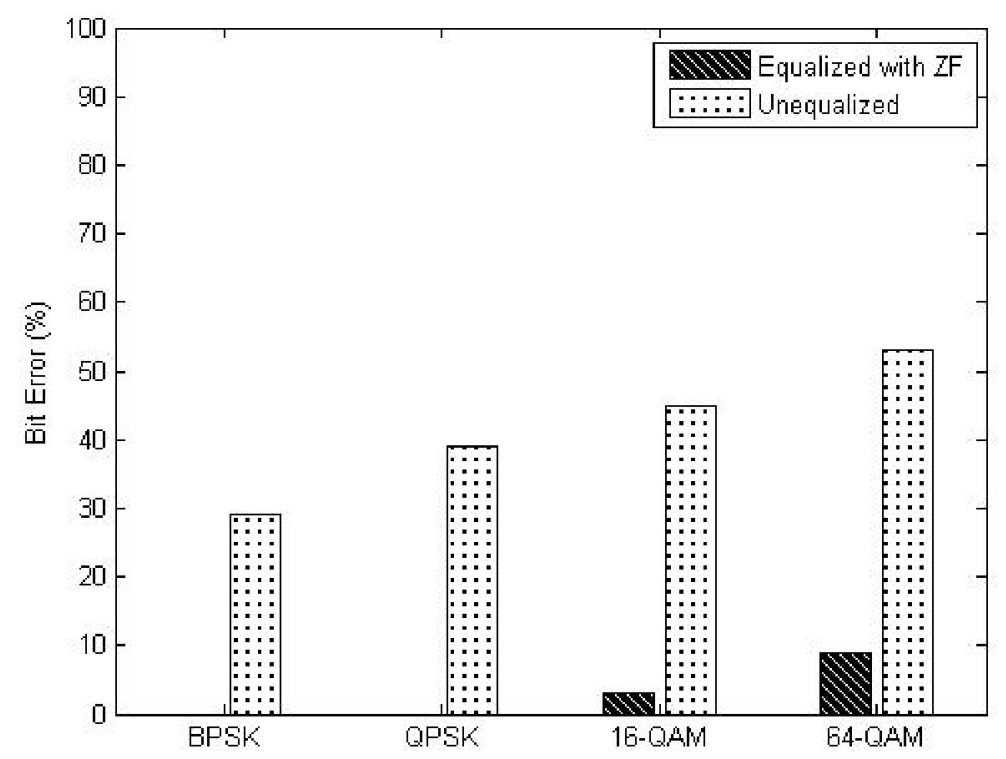

Figure 7. The compairing of bit error for unequalized data and use ZF equalizer

\section{CONCLUSION AND FUTURE WORK}

A LabVIEW application has been developed to implement OFDM communication system use BPSK, QPSK, 16-QAM and 64-QAM modulation. Moreover, $\mathrm{ZF}$ equalizer is employed to achieve reliable system. The IQ rate determine the bandwidth available, but in this experiment good performance is achieved with IQ rate less than $1 \mathrm{MHz}$. BPSK, QPSK and 16-QAM are more resistant with noise enviroment than 64-QAM. The good performance of ZF equalizer is achieved when is modulated using BPSK, QPSK and 16-QAM. By applying ZF equalizer can reduce bit error from $29,16 \%$ and $39,06 \%$ into $0 \%$ when use BPSK and QPSK modulation. Whereas, the bit error can be pressed became 3,125\% and 8,85\% when 16-QAM and 64-QAM modulation be used. Next experiment will constructed OFDM PHY scheme with MMSE equalizer to increase the reliability OFDM system which be implemented use USRP as RF front end.

\section{REFERENCES}

[1] Y. Sun,Bandwidth-Efficient Wireless OFDM, IEEE Journal on selected area in Communication, Vol. 19, No. 11, November 2001.

[2] M. Ergen,Mobile Broadband Including WiMAX and LTE, pp109, ISBN: 978-0-387-68189-4, New York, USA, Springer Science+Business Media, LLC 2009.

[3] S. Costanzo, F. Spadafora, A. Borgia, H. O. Moreno, A. Costanzo, G. Di Massa, High Resolution Software Defined Radar System for Target Detection, Journal of Electrical and Computer Engineering, Volume 2013.

[4] A. Marwanto, M. A. Sarijari, N. Fisal, S. K. S. Yusof, R. A. Rashid, Experimental Study of OFDM Implementation Utilizing GNU Radio 
and USRP-SDR, Procceding of the 2009 IEEE $9^{\text {th }}$ Malaysia International Conference on Communication, pp. 132-135,ISSN:978-1-4244-5532-4, Kuala Lumpur, Malaysia, 15-17 December 2009.

[5] Abirami M, Gandhiraj R, Soman K. P, Performance Analysis of Real Time OFDM Based Communication System Using GNU Radio and USRP, International Journal of Advanced Research in Computer Science and Software Engineering, ISSN : 2277 128X, Volume 3, June 2013.

[6] A. Y. F. Youssef, K. M. H. Hassan, M. G. Mostafa, M. T. Saad, M. Khairy,Implementation of a Wireless OFDM System using USRP2 and USRP N210 Kits,Graduation Project Report,Faculty of Engineering,Cairo University,Giza,Egypt,July 2012.

[7] I. Kardaras, A. Liavas,Software-Defined Radio Implementation of an OFDM Link, Master of Science, Technical University of Crete, Department of Electronic and Computer Engineering,2010.

[8] A. Kaschub, C. Muller, M. Proebster, T. Werthmann, M. Schmidt,Design and Implementation of an OFDM-based Communication System for the GNU Radio Platform,Master Thesis, Institut fur Kommunikationsnetze und Rechnersysteme,Universitat Stuttgart.Germany,2009.

[9] G. Berardinelli, P. Zetterberg, 0. Tonelli, An SDR Architecture for OFDM Transmission over USRP2 Boards, Signals, Systems andComputers IEEE Conference, pp 965-969, ISSN : 1058-6393, Pacific Grove, CA,6-9 Nov 2011.

[10] A. Kanchan, S. Dwivedi, Comparison of BER Performance in OFDM Using Different Equalization Techniques, International Journal of Engineering and Advanced Technology (IJEAT), ISSN:2249-8958, Volume-1, Issue-6,August 2012.

[11] S. Ohno,Performance of Zero-Forcing Equalizer for Single-Carrier Zero-Padded Transmissions Over Multipath Fading Channels,IEEE International Conference onAcoustics, Speech, and Signal Processing(ICASSP '04), pp IV-1017-IV-1020,ISSN:1520-6149,2004.

[12] A. Zainudin, A. Sudarsono, I. G. P. Astawa,Reliability Analysis of Digital Communication for Various Data Types Transmission using GNU Radio and USRP, Industrial Electronic Seminar (IES) 2013,pp 91-96, ISBN 978-602-9494-87-7, Electronic Engineering Polytechnic Institute of Surabaya,2013.

[13] A. Gupta, A. Forenza, R. W. Hearth Jr.,Rapid MIMO-OFDM Software Defined Radio System Prototyping, pp 182-187, Signal Processing System IEEE Conference,2004.

[14] E. Grayver,Implementing Software Defined Radio,pp 145,Springer Science + Business Media, New York,2013.

[15] Ettus Research Data sheet, USRP, USRP N210, http:www.ettus.com, accessible April, 2014.

[16] National Instrument Data sheet, NI USRP-2920, NI USRP-2921, http://indonesia.ni.com, accessible April, 2014. 